\title{
Tentatives et tentations de la poésie face au temps
}

Postpoésie et poésie fractale

Attempts and Temptations of Poetry in the Face of Time. Postpoetry and Fractal Poetry

\section{David Gondar}

\section{QpenEdition Journals}

Édition électronique

URL : https://journals.openedition.org/cher/4464

DOI : $10.4000 /$ cher.4464

ISSN : 2803-5992

\section{Éditeur}

Presses universitaires de Strasbourg

\section{Édition imprimée}

Date de publication : 30 novembre 2021

Pagination : 133-150

ISBN : 979-10-344-0095-9

ISSN : 1968-035X

\section{Référence électronique}

David Gondar, «Tentatives et tentations de la poésie face au temps », reCHERches [En ligne], 27 | 2021, mis en ligne le 30 novembre 2021, consulté le 12 janvier 2022. URL : http://journals.openedition.org/ cher/4464; DOI : https://doi.org/10.4000/cher.4464

\section{(c) (i) (2)}

Ce(tte) œuvre est mise à disposition selon les termes de la Licence Creative Commons Attribution -

Pas d'Utilisation Commerciale - Partage dans les Mêmes Conditions 4.0 International. 


\title{
Tentatives et tentations de la poésie face au temps Postpoésie et poésie fractale
}

\author{
DAVID GONDAR
}

Bis repetita placent Horace, Art poétique

\begin{abstract}
$\ll \int^{\mathrm{e}}$ e traduis, tu traduis, nous traduisons...", écriture et lecture sont des interprétations aux variables exponentielles. Composer, construire un texte dans le but, la recherche, l'attente d'un lecteur, est un procédé dépendant du genre, du format, du lieu et de l'époque. Si le texte, qu'il soit littéraire, scientifique ou commercial, ne se conçoit pas selon les mêmes préceptes, la lecture est, elle aussi, sensible à des facteurs multiples selon les objectifs du message et du récepteur. Lorsque «je» lis un texte, j'amène avec moi des bagages linguistiques, culturels, intimes dans un exercice d'appropriation, de récupération. Il en va de même lorsque l'auteur écrit, «il» a pour tâche d'élaborer un texte codifié qui connaîtra plusieurs lectures et relectures, toutes dépendantes d'un lecteur qui procédera ainsi à une réécriture de l'œuvre première. Si l'auteur pose la première pierre, œuvrant à l'édification d'un message, littéraire ou non, c'est le lecteur qui endosse un rôle indivisible de lecteur-auteur. Lire c'est donc dire d'une voix unique ou dans une tentative d'imitation, de répétition. Si l'intelligence artificielle propose de nouvelles spéculations sur la définition du sensible, du vivant, du penseur; la machine, à travers de nombreuses expérimentations scientifiques ou artistiques, remplace l'auteur d'os et de chair, dans la création de messages reposant sur des algorithmes, des dictionnaires, des limites. La machine s'ouvre à l'acte de création par l'homme, mais elle trouve ensuite une autonomie grâce à l'informatique ou la robotique. L'homme écrit la machine
\end{abstract}

* David Gondar, enseignant d'espagnol, spécialiste de poésie hispanique contemporaine, Université de Strasbourg, faculté des Langues et Sciences Po, CHER UR 4376 et Écoles d'ingénieurs (INSA, ENGEES, EOST, TPS et ECAM), . 
et lit la machine. Ce "nous», homme et machine, englobe écriture et lecture, vertigineuse mise en abîme de nœuds dans la conception. Qui émet le message?

La simultanéité évoquée par une lecture-réécriture fonctionne par un réseau de nœuds interpersonnels. Le déplacement du message d'un hypo- à un hyper- est l'ajout de soi, "nous traduisons». L'effacement de nos frontières culturelles, politiques et intimes établit des dérivations du texte, des réceptions transpersonnelles, s'écoulant de l'un à l'autre, muant le lecteur en auteur, le temps de la lecture en temps de l'écriture. L'auteur révoqué devient-il alors lecteur à la découverte de son texte dans la bouche d'un autre? Peut-on s'éloigner jusqu'à des rives où l'oubli est familier? "je» serait un "tu», sensation trompeuse de proximité sans pour autant atteindre l'intime. "Tu traduis» délaisse le «nous», présence/absence: «je te sais lecteur et pourtant tu me dis».

Blaise Pascal annonce dans De l'esprit géométrique (1896) que le temps est une notion «qu'il est impossible et inutile de définir» (p. 116). Il poursuit:

Qui le pourra définir? Et pourquoi l'entreprendre, puisque tous les hommes conçoivent ce qu'on veut dire en parlant de temps, sans qu'on le désigne davantage? Cependant il y a bien de différentes opinions touchant l'essence du temps. Les uns disent que c'est le mouvement d'une chose créée; les autres, la mesure du mouvement, etc. Aussi ce n'est pas la nature de ces choses que je dis qui est connue de tous: ce n'est simplement que le rapport entre le nom et la chose; en sorte qu'à cette expression, temps, tous portent la pensée vers le même objet: ce qui suffit pour faire que ce terme n'ait pas besoin d'être défini, quoique ensuite, en examinant ce que c'est que le temps, on vienne à différer de sentiment après s'être mis à y penser; car les définitions ne sont faites que pour désigner les choses que l'on nomme, et non pas pour en montrer la nature.

Le mot «temps» provient du latin tempus (temps, fraction de la durée) lorsqu'il indique époques, moments; mais pour désigner le temps en rhétorique ou en poétique, il lui sera préféré le grec khronos. Précisons que le mot «temps" comporte à la fois les valeurs du tempus et de l'aevus, autrement dit les notions de «fractions de durée» et de «durée continue». Concevoir le temps, c'est concevoir les mouvements du passé, du présent et du futur. La présentation du 13 mars 2018 de la psychiatre Anne Giersch, directrice de l'unité INSERM 1114, conférence ouvrant le cylce de séminaires du laboratoire CHER UR4376 et ayant pour titre: "La perception du temps chez les patients souffrant de schizophrénie ", accentuait l'importance dans la notion du temps de l'avant et de l'après. Si en musique, la mélodie donne à l'auditeur les informations nécessaires de la note jouée en lien avec celle qui précède et celle qui suit, autrement dit un présent connecté au passé et au futur, nous pouvons reprendre ce même exemple pour l'image en nous appuyant sur la photographie, dont l'élément figé raconte un avant et un après également. Arnaud Claass dans Du temps dans la photographie (2014: 9), remarque: «il y a une éloquence de la mobilité dans le mutisme de l'image fixe». Il oppose l'inertie de l'instant photographique à la fuite du temps. La décomposition-recomposition des phénomènes perceptifs, développés par les chercheurs de la Gestalttheorie, ainsi que par les sciences 
cognitives, comme pour la psychophysiologie de la perception visuelle, indique que le rapport établi avec l'image (ou avec le cliché photographique), n'est pas dans l'instantané, le "fractionnement interstitiel» (la fragmentation) mais dans une recomposition, une réécriture, dont l'œil et le cerveau comblent les brèches, assemblent les pièces du puzzle spatio-temporel. Claass indique également à la page 62 :

Chaque photographie est une fabrique d'espace-temps, organisée d'une certaine manière, de façon à construire, même instantanément, une proposition, une "phrase», en fonction d'un horizon de sens, c'est- à-dire une unité susceptible de rendre le monde intelligible.

Mais il y a aussi dans le temps, le Dasein de Heidegger, l'existence et la présence, l'«être ici», cher au poète espagnol Jorge Riechmann. L'essence est par définition hors du temps, alors que l'existence, pour reprendre Aristote, est la persévérance dans le temps, la "puissance mise en acte». Dérivant du latin existere, l'existence signifie "sortir de», "procéder». Pour Jean-Paul Sartre, «l'existence précède l'essence» (1996: 26).

Nous allons aborder le temps dans la poésie espagnole actuelle à travers la «Postpoésie» mise en opposition par le poète, essayiste et scientifique Agustín Fernández Mallo à la "poésie orthodoxe», nommée également "poésie de la normalité» par le poète et critique Vicente Luis Mora. Puis, nous poursuivrons par l'étude de la poésie fractale de Ramón Dachs et ses six écritures géométriques. Les tentatives de ces poétiques cherchent à établir un espace topologique où des réseaux inespérés admettraient et hébergeraient l'émancipation des fondations littéraires dans un temps anhistorique. Les tentations d'une poésie de l'effacement ne risquent-elles pas de produire des supernovas poétiques? Si l'espace et le temps viennent à se fondre dans une poésie du post- et une écriture fragmentée, est-il à craindre que ces explosions n'engendrent des trous noirs littéraires, une poésie du spam?

\section{«Postpoésie » et «système orthodoxe »}

La poésie espagnole actuelle est en pleine mutation de formes et de sens. Les poètes se regroupent autour d'enjeux similaires: refonte des mécanismes, multiplication des systèmes poétiques, décomposition des références temporelles. Dès 2000, Agustín Fernández Mallo pose le concept de «Poésie Postpoésie» (Poesía Postpoesía) et le développera dans des articles publiés dans des revues comme Contrastes, Lateral ou Quimera. Il le reprendra également dans son «poemario-performance» Creta lateral traveling (2004), en guise de conclusion. Il s'agit de déconstruire la poésie car elle est la dernière discipline artistique à ne pas l'avoir fait selon l'auteur. Jean-Christophe Bailly dans son essai L'élargissement du poème (2015: 53) niera ce constat: «le poème est à la fois la forme littéraire la plus ancienne et celle qui est le lieu le plus actif du renouvellement formel». Vicente Luis Mora insiste lui sur la "perpétuelle mutation» de la poésie et du 
poète dans Singularidades (2006: 118). Si Fernández Mallo définit la «Poésie postpoétique» ou "Poésie étendue» (Poesía expandida) en opposition avec ce qu'il désigne comme la "Poésie orthodoxe», c'est afin de rompre avec toute fondation. L'hybridation est présente dans la "postpoésie» dans le refus des classifications génériques. Poème et essai, théorie et pratique, sont combinés pour mieux effacer les frontières habituelles. Le «Système Poétique Orthodoxe» lorsqu'il utilise des influences étrangères à l'art, ne le fait que dans un acte d'effacement du non-poétique. C'est une poésie «référence» où ce qui provient de l'extérieur n'est admis qu'après conversion aux dogmes poétiques établis. La poésie est donc «isolée» ou «fermée» selon la terminologie de Fernández Mallo. Le seul échange possible avec ce qui l'entoure est de caractère énergétique et non matériel. Les références extérieures que s'accapare la "Poésie Orthodoxe», dans un réseau "fermé », sont en fonction de la force centripète et se retrouvent attirées vers le centre de la trajectoire. Elles finissent par y demeurer, ne pouvant alors plus se modifier ni évoluer. Par conséquent, elles meurent. Dans le cas d'un réseau "isolé», n'ayant d'échange ni d'énergie ni de matière, elles finissent par exploser en raison de leurs propres poids et énergie. Fernández Mallo nomme cela l'«effet de serre poétique» (efecto invernadero poético) car le "Système Poétique Orthodoxe» admet des entrées mais pas de sorties (des références). L'accumulation d'éléments extérieurs est par la suite transformée, suscitant un échange d'énergie vers l'intérieur. Ce système ne renvoie pas d'énergie et encore moins de matière vers l'extérieur. Il est un réseau fermé à une seule voie sans issue. Recourant à une comparaison empruntée à la circulation routière, l'auteur évoque la conséquence d'un système «désorganisé». C'est le sens de l'organisation des automobilistes qui permet de mettre fin aux embouteillages. Mais dans le cas du "Système Poétique Orthodoxe», le manque d'organisation dû aux règles explicites ou implicites, tabous et pratiques, empêchent à la poésie d'évoluer: "es más, hoy por hoy, tal como se practica, hay que decir claramente que es el sistema artístico más rígido e hipertrofiado que existe ${ }^{1}$ » (Postpoesía: 149). Pour l'auteur, tout type de poésie se constitue en réseau. Le réseau de type "Poésie Orthodoxe» comprend peu de nœuds très connectés et un grand nombre de nœuds très faiblement connectés. Donc si un nœud considérablement connecté s'annule, ce sont aussi toutes ses liaisons qui se voient annulées. L'auteur le nomme l'«habitat du système poétique».

\section{Attracteur "poésie orthodoxe » : zone stable et non-chaotique}

La notion d' "attracteur" est empruntée aux systèmes classiques de Newton ou de la Relativité et se définit par un système non-chaotique et un temps inexistant.

1 «de plus, actuellement, de la manière dont elle est pratiquée, il faut dire clairement que c'est le système artistique le plus rigide et hypertrophié qui existe». Nous traduisons l'ensemble des citations de langue espagnole. 


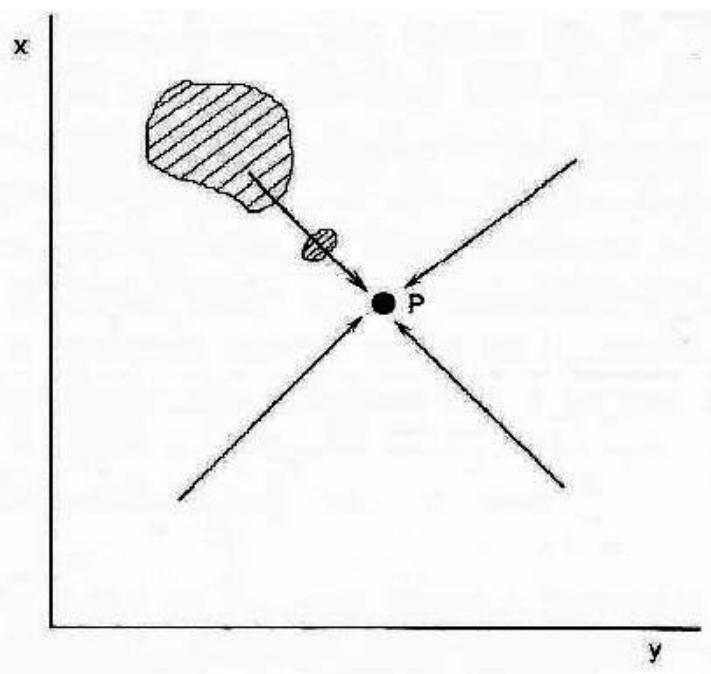

Sean cuales sean las condiciones iniciales el sistema evolucionará necesariamente hacia el Atractor P.

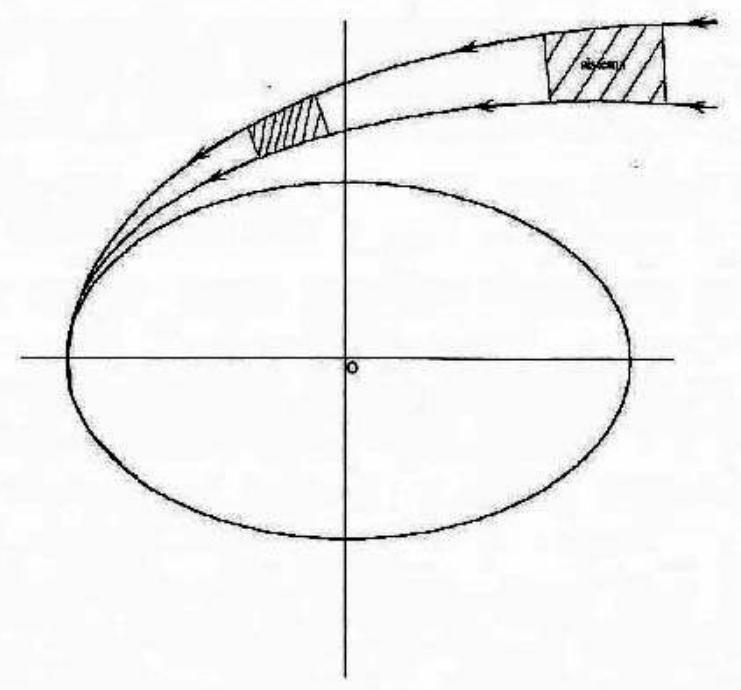

Evolución del sistema hacia un atractor lineal y estable tipo elipse.

Postpoesia, @ Agustin Fernandez Mallo.

La première illustration (Postpoesía: 167) nous montre que le système ou l'objet, évolue toujours vers l'attracteur. Comme dans le cas des réseaux fermés, le mouvement va de l'extérieur vers l'intérieur: l'objet est donc toujours pris dans un mouvement vers l'attracteur. Dans la seconde illustration, le mouvement se 
fait vers l'attracteur non plus en ligne droite mais à partir d'une courbe elliptique. Si l'objet s'écarte de la courbe, il finit toujours par retrouver sa trajectoire. C'est pourquoi le temps n'existe pas car, quoi qu'il arrive, le système retrouve sa forme initiale. Si le «Système Poésie Orthodoxe» admet des entrées mais aucune sortie, il est alors un trou noir selon la sentence de Fernández Mallo.

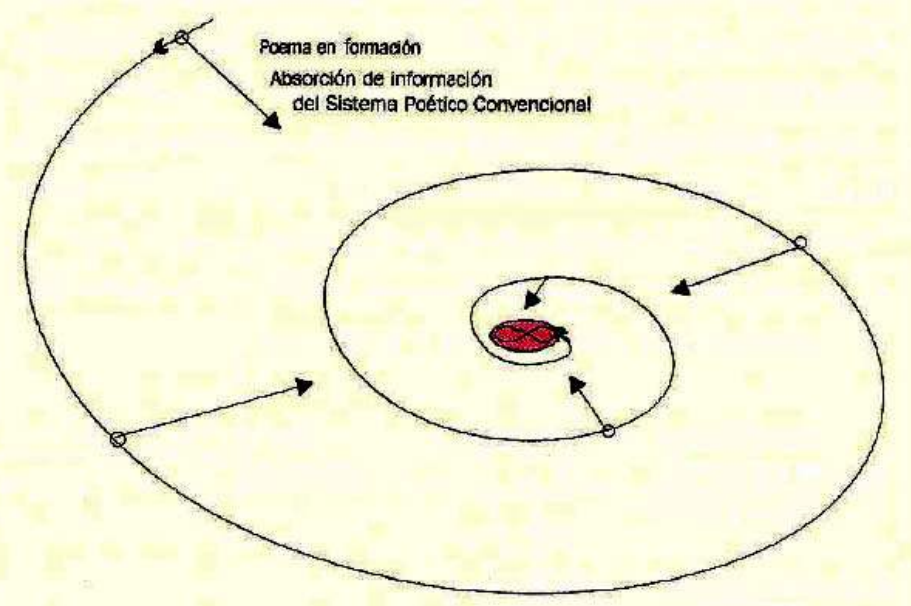

El círculo central constituye la Red Sistema Poético Convencional. El poema en formación, atraído por la absorción cada vez mayor de información, cae a medida que se hace, y una vez finalizado permanece encerrado en ese centro alimentando a otros poemas de su mismo entorno: sistema en continuo equilibrio.

Postpoesia, (c) Agustin Fernandez Mallo.

L'illustration, ci-dessus (2009: 170), indique l'absorption du poème conventionnel par un mouvement spiroïdal jusqu'à l'attracteur au centre. Il n'y a pas d'évasion, le poème en formation finit par échouer dans un système assimilateur où tout est lié au "Système Poésie Orthodoxe» par la forme et les propositions. Linda Lê développe un concept similaire au «rhizome postpoétique» de Fernández Mallo dans son article «Littérature déplacée» (1999: 330), à partir de l'image de la plante adventive, mais appliqué à l'exil.

Nous glissons ainsi du premier sens de la «littérature déplacée», entendue comme «paroles d'exil», au deuxième sens, celui d'une littérature qui ne trouve pas sa place. Une littérature qui serait comme une plante adventive. Ni d'ailleurs ni d'ici. Et, comme telle, elle perturbe l'ordre naturel des choses. Elle cherche à rompre avec l'autorité.

Il s'agit bien chez Linda Lê, comme chez Fernández Mallo, d'une littérature inattendue, ou pour reprendre l'image de la botanique, une sorte de plante adventive qui regreffe ici ou ailleurs, un bouturage étrange et accidentel. La 
littérature déplacée naît de telles excroissances inhabituelles ou supplémentaires, adventives. La «Poésie Orthodoxe» fait le choix d'un discours simple qui facilite la compréhension de l'œuvre dès la première lecture. Comme le soulignait le critique Daniel Bellón, cela évite les relectures. Si les poèmes doivent compter entre douze et soixante vers, le recueil doit quant à lui se composer de vingt à soixante poèmes. Euvre courte, permettant une lecture rapide, ce type de poésie est à l'opposé d'une «littérature de l'effort» développée par le "système postpoétique».

\section{«Système postpoétique »}

La «Poésie Postpoésie» reprend de la «Poésie de la différence» (Poesía de la diferencia), "Poésie du je» (Poesía del yo) ou "Poésie du silence» (Poesía del silencio), l'expérimentation, comme chez José Ángel Valente, María Victoria Atencia ou Antonio Gamoneda, mais elle n'est pas particulièrement attachée à un genre. Elle est perméable aux différentes poétiques, impérialiste et aspire à faire feu de tout bois. La mutation que recherche Fernández Mallo est celle qui permet au poète d'être en perpétuel mouvement. Nous utilisons le terme de «mouvement perpétuel» plus dans un sens musical que scientifique. Car dans le cas du mouvement perpétuel mécanique, le système n'existe que par luimême sans apport extérieur d'énergie ou de matière. Alors que la "Postpoésie» est une création générée par l'échange, elle requiert des éléments extérieurs pour se construire; à l'inverse de la "Poésie Orthodoxe» qui peut être isolée et par conséquent fonctionner en réseau fermé. Le mouvement perpétuel dans la musique ou moto perpetuo est davantage représentatif des intentions de la "Postpoésie» car il utilise la continuité de la mélodie afin d’y introduire la répétition. La reprise musicale est l'espace où se mêlent les notes d'une mélodie en cours à une autre naissante dans un tempo rapide. Fernández Mallo mentionne Paul Virilio afin de souligner que l'art ne cherche de repères temporels ni passés ni futurs, mais devient «l'instrument privilégié du présent et de la simultanéité». Dans la «Postpoésie», le mouvement perpétuel marque l'intersection des éléments dans la création. Il s'agit de trouver une nouvelle architecture à la poésie. Au «Il faut être absolument moderne» d’Arthur Rimbaud, Fernández Mallo surenchérit par "hay que ser «relativamente posmodernos»" («il faut être «relativement postmoderne»»). Shoshana Felman dit au sujet de Rimbaud: "l'absolument moderne est ce qui manque à être», ce "je est un autre» de sa lettre adressée à Georges Izambard du 13 mai 1871. Car la clef réside dans cette absence, ce qui reste à définir et c'est cela que la «Postpoésie» tente de proposer comme édification: la définition $\mathrm{du}$ «je» par ce qui l'entoure: société, culture et science, dans une toile tissée à partir du présent tout en englobant le passé ainsi que le futur. Si Rimbaud utilise le «je» comme une troisième personne afin de créer une prise de distance, ce «je» est "l'absolument moderne», et cet «autre» est «ce qui manque à être». On ne peut définir le «je» que par «l'autre». La postmodernité évoquée par Fernández Mallo n’existe que par une 
définition de soi à travers son entourage. Ce «je» est alors à l'autre car c'est lui qui comble le manque et définit l'absence. Antonio Portela dans son poème au titre évocateur «Hay que ser absolutamente posmoderno» ( Il faut absolument être postmoderne») (2003: 53) développe certains points très similaires.

Nous ne sommes plus dans le «relativement» de Fernández Mallo au ton décalé, ironique comme il l'explique (Postpoesía: 48-49):

Hoy por hoy, ya no creemos ni en nosotros mismos, no nos tomamos en serio, tenemos la convicción de que todo producto artístico es ridículo si no lleva dentro de sí su propia frustración, si no articula una parodia o una caricatura de sí mismo².

Il y a dans la «Postpoésie» une distance, un décalage invitant à considérer l'œuvre au second degré. Portela en revanche, est dans l'absolu rimbaldien. Il est le pont entre Rimbaud et Fernández Mallo, il unit l'absolu au relatif et le moderne au postmoderne. Dans son poème «Hay que ser absolutamente posmoderno », Portela écrit dans la première strophe:

Hay que ser absolutamente posmoderno.

Simultáneo a cada hombre del presente,

a todas las edades de la Historia,

mi memoria se fragmenta ${ }^{3}$.

À la référence rimbaldienne, ces trois vers ajoutent l'idée d'une simultanéité. Nous retrouvons ici le temps multiple concentré dans le présent à travers chaque homme et chaque époque de l'Histoire. Avec la fragmentation de la mémoire, nous ne sommes par ailleurs pas si loin de la fragmentation informatique.

Yo y mis contemporáneos

hemos aprendido el olvido.

Estoy libre del pasado y juego con él:

descanso con otras formas eternas ${ }^{4}$.

Dans cette strophe, Portela s'éloigne de Rimbaud. Il y identifie son groupe (ses contemporains et lui) et son pouvoir de faire abstraction du passé (nous avons appris l'oubli). Libéré du passé, le poète peut s'en servir à son gré.

Puedo elegir mi tiempo. No así mi espacio.

Mi vida y mi cultura se componen

de formas de calidoscopio.

Nunca fuimos tan libres 5 .

2 «Actuellement, nous ne croyons plus en nous-mêmes et nous ne nous prenons, pas plus, au sérieux; nous avons la conviction de que tout produit artistique est ridicule s'il ne comprend pas en son sein sa propre frustration, et s'il ne conçoit pas sa propre parodie ou caricature.»

3 «Il faut absolument être postmoderne. / Simultané à chaque homme du présent, / à toutes les périodes de l'Histoire, / ma mémoire se fragmente.»

4 «Moi et mes contemporains / avons appris l’oubli. / Je suis libéré du passé et joue avec lui : / je repose avec d'autres formes éternelles.»

5 «Je peux choisir mon temps. Et non mon espace. / Ma vie et ma culture se composent / de formes de kaléidoscope. / Nous ne fûmes jamais si libres.» 
Dans l'image du kaléidoscope, nous retrouvons l'apport de l'extérieur (vie et culture) sur la poésie. Si le poète peut faire du présent le point où se concentre toute l'Histoire, ce présent a un enracinement culturel et social qui se répercute sur la poésie. Le poète décrit un temps kaléidoscope, fluide, flexible, qui joue des simultanéités, des juxtapositions, du mouvement ondulatoire, qui fait osciller entre oubli et mémoire, disparition et surgissement.

Es el comienzo de una nueva era menos novedosa que las anteriores. Mi momento es el resumen de todos los momentos del mundo ${ }^{6}$.

Le poème se termine par ces vers, où il réinscrit la «Postpoésie» dans l'Histoire de la poésie et notamment dans la continuité des innovations de la Modernité et, de manière plus étendue, des avant-gardes qui ont parcouru le $\mathrm{xx}^{\mathrm{e}}$ siècle. Il revient une dernière fois sur la concentration du temps en un point - ici «mi momento» (mon instant), concentration des temps, présent, passé, futur, mais aussi du temps intime, personnel et du temps universel. L'instant du poète est le noyau où se concentre toute l'histoire du monde: le poète est acte d'universalité atemporelle et apatride, à la fois mouvement centrifuge et centripète, l'un ouvrant le poème, l'autre le fermant. Les derniers vers reprennent cette idée de temps englobant et englobé, au cœur d'une nébuleuse humaine, historique, planétaire, un noyau dans lequel converge cette totalité, des temps parallèles qui aboutissent en un point unique où ils interagissent.

\section{Une poésie du feedback dans un réseau ouvert}

La représentation, page suivante (2009: 150), montre comment, par opposition au système fermé de la «Poésie Orthodoxe», la «Postpoésie» échange avec les références extérieures. Elle n'est pas seulement perméable, elle se laisse aussi absorber. Il y a donc un flux à double sens (intérieur et extérieur) à l'origine de trois types de mouvements: premièrement, s'ajoute à la force centripète une force centrifuge. La force centripète n'a pas le même effet que dans le «Système Poésie Orthodoxe» car les informations ne pouvant pas stagner, elles ne meurent pas. Quant à la force centrifuge au lieu d'attirer les références vers le centre, elle provoque une rotation qui les en éloigne. C'est ainsi que les informations circulent de l'extérieur vers l'intérieur et viceversa. Deuxièmement, Le «Système Postpoétique» est un réseau ouvert plat, il ne comporte ni haut ni bas, ni intérieur ni extérieur. Il est comme le précise Fernández Mallo: "virtualmente infinito: un mapa» («virtuellement infini : une carte») (2009: 151). C’est le rhizome emprunté à la botanique par Deleuze et Guattari. Le rhizome est cette plante qui pousse sur un plan horizontal et sans racine, bonne pour l'aération des sols et utilisant la multiplication végétative.

6 «C'est le commencement d'une nouvelle ère / moins innovante que les précédentes. / Mon instant est le résumé / de tous les instants du monde.» 


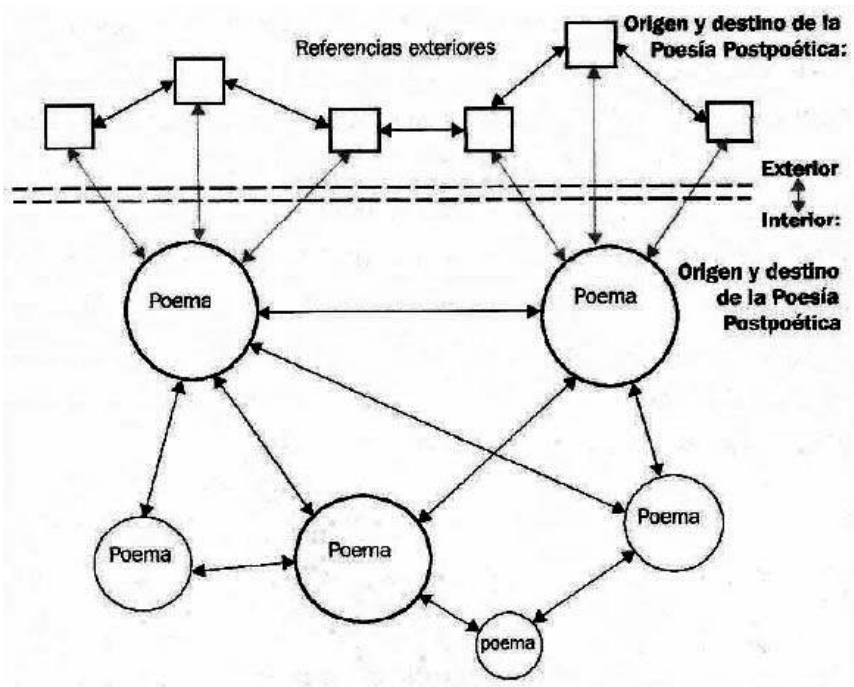

Representación del Sistema Postpoético como una Red Abierta.

Postpoesia, ( ) Agustin Fernandez Mallo.

Troisièmement, le "Système Postpoétique» s'auto-organise de manière spontanée et immédiate. Il y a donc quelques similitudes avec la mauvaise herbe puisque la «Postpoésie» se développe aussi par la multiplication végétative qui:

- remet à zéro les cellules de la plante qui ne connaissent alors pas de patrimoine génétique. Il s'agit de se défaire des figures poétiques classiques, des styles et des formes archaïques.

- envahit rapidement les sols par le clonage. La «Postpoésie» se développe très rapidement par les intertextualités et les hypertextes ou l'utilisation d'œuvres existantes (comme El hacedor (de Borges), Remake de Fernández Mallo).

Parmi les risques, existent les pathogènes strictes ou opportunistes. Les infections proviendraient alors soit d'auteurs et d'œuvres saines qui ont un sens et une place dans l'Histoire littéraire, soit d'auteurs ou d'œuvres malades ou vieillissantes qui ne s'articulent plus avec la même justesse. Le risque de disparition peut être contré par la protection et la sauvegarde d'un système construit en réseau ouvert où les nœuds de connexion ne sont pas les poètes, écoles poétiques ou institutions comme dans la "Poésie Orthodoxe», mais les œuvres, les poèmes (2009: 162-163):

[...] esta red posea atributos de entidad apersonal: la Postpoesía es Opus-céntrica en lugar de Tribal-céntrica, los nodos son las obras, no las escuelas, obras que 
tienden lazos a otras obras, sean éstas de la escuela que sean, incluidas las de la poesía ortodoxa ${ }^{7}$.

La «Postpoésie» a donc établi un réseau de connexions en nœuds plus ou moins forts. Les plus forts comme nous l'avons vu sont les œuvres, le noyau du mouvement. Les nœuds moyens sont les éléments et les références qui s'ajoutent au poème aux niveaux social, scientifique ou culturel. Viennent ensuite les nœuds les plus faiblement connectés, c'est-à-dire ceux qui définissent la «Poésie Orthodoxe» : poètes, écoles poétiques, institutions.

\section{Attracteur "poésie postpoésie»: zone instable et chaotique}

Si le «Système Orthodoxe» s'appuyait sur des attracteurs stables, la «Postpoésie» est au contraire dans une zone instable où, comme nous l'avons vu pour le cas du rhizome, il n'y a pas de racine ni de plan vertical mais seulement un plan horizontal. De plus, la perméabilité du réseau aux éléments contemporains de la société actuelle et le va-et-vient entre la "Postpoésie» et la société ainsi que les différents degrés de nœuds connectés font de ce système un territoire instable propice au chaos où les attracteurs provoquant des trajectoires imprévisibles et donc incalculables. Les attracteurs "étranges», comme dans le système dynamique différentiel de Lorenz, utilisent une trajectoire erratique en forme d'ailes de papillon. Ce chaos n'est donc pas un désordre mais un système complexe dans lequel convergent des éléments habituellement divergents.

\section{«Pangée » et la «poésie de la normalité »}

Le concept de «Pangée» développé par Vicente Luis Mora se réfère à un monde où, à nouveau, les cinq continents sont réunis pour ne former plus qu'un. La totalité des terres émergées rassemblées sont chez Mora le cyberespace. Les mutations poétiques utilisent alors l'informatique pour offrir de nouvelles propositions. De la poésie assistée par ordinateur comme chez Eugenio Tisselli (El drama del lavaplatos: 2010) aux publications digitales, il y a, comme le souligne Mora, un questionnement sur le changement de paradigme. L'écran fictionnel de l'ordinateur interroge sur la valeur du cadre et de l'objet mais aussi sur le lecteur qui devient de plus en plus un spectateur. Dans le panorama actuel des nouvelles technologies, le poète doit maîtriser les nouvelles techniques afin de rester connecté au monde qui l'entoure, non dans un élan poétique mais à des fins de création et de visibilité. En opposition avec la «normalité» poétique, nous allons voir comment Mora définit les critères d'une poésie innovante, une poésie des singularités. Si le microrécit par sa brièveté, sa rapidité et sa fragmentation incarne une littérature hypermoderne, les similitudes avec

7 «[...] ce réseau possède les attributs de l'entité apersonnelle: la Postpoésie est Opuscentrique au lieu de Tribal-centrique; les nœuds sont l'œuvre, non les écoles, des œuvres qui tissent des liens vers d'autres œuvres, peu importe les écoles, en incluant celles de la poésie orthodoxe.» 
l'aphorisme et le haïku indiquent aussi une présence contemporaine de la poésie dans le quotidien (publicités, slogans, discours politiques). L'image du texte digital comme un palimpseste où l'on peut effacer et réécrire à l'infini expose le concept d'une littérature éphémère existant à travers ses mutations.

La «Poésie de la normalité» (Mora) et la "Poésie orthodoxe» (Fernández Mallo) s'opposent à la «Poésie Postpoésie» (Fernández Mallo) et à la «Poétique des singularités» (Mora). Pour définir la «Poétique des singularités», il faut la concevoir comme un regroupement de formes qui n'ont trouvé à s'intégrer dans aucun mouvement. Mora propose de constituer une anthologie dont le titre serait Excluidos. Poetas al margen de la tradición historiográfica española (1920-1999)8. On y retrouverait les poètes qui n'ont su s'inscrire ni dans leur temps ni dans les mouvements existants. Parmi eux, il y aurait entre autres: Gamoneda, Valente, Ángel Crespo, César Simón, Luis Feria, Chicharro, Carlos Edmundo de Ory, los Padorno, Carriedo, María Victoria Atencia, Francisco Pino, Joan Brossa, Quiñónez, Pérez Estrada, et Fonollosa². Cette «singularité» poétique s'apparente à l'«atopia» dont l'écrivain et critique Éric Bonnargent nous dit qu'elle est «sans lieu» (2011: 14), désignant celui qui n'est pas dedans, ou à la "littérature des exceptions» (literatura de las excepciones) d'Andrés Sánchez Robayna qui, pour sa part, parle d’une poésie de «lieu sans lieu» («lugar sin lugar»), d'une littérature de «solitaires, d'exceptions» («de solitarios, de excepciones»). La "Poésie de la singularité» est à la recherche d'un espace en-dehors, en-delà, des lieux convenus de la "poésie de la normalité». Elle est une poétique du Puer Senex, en ce que le poète réussit à s'inscrire dans son temps tout en y étant anachronique. Comme le disait Mora: Cervantès, Joyce, Picasso ou Kubrick ne sont pas les produits du darwinisme mais des ovnis ne répondant à aucune suite logique. Il y a dans la conception de la «Poétique des singularités », un regroupement par deux critères principaux: l'originalité et la marge. Les listes ou les catégories sont à exclure car elles enferment les poétiques "singulières", donc distinctes, dans des groupes identiques.

Fernández Mallo et Mora distinguent la poésie actuelle en mutation de la «poésie orthodoxe» ou "poésie de la normalité». Mais, ils ne rejettent pas pour autant l'influence exercée sur leurs œuvres par des poétiques antérieures. La réécriture "postpoétique» est un acte d'appropriation qui ingère œuvres passées et présentes mais également récupère des éléments externes à la poésie et à la littérature pour établir un dialogue. Ce dépassement extralittéraire élargit le public en construisant de nouvelles versions et des renouvellements, un multivers convoquant un espace-temps malléable.

8 Mora publiera en 2016 l'anthologie de poésie La cuarta persona del plural (19782015) regroupant les 22 poètes:Rikardo Arregi, José Ángel Cilleruelo, Jesús Aguado, Esperanza López Parada, Eduardo Moga, Jorge Riechmann, Vicente Valero, Diego Doncel, Ada Salas, Álvaro García, Jordi Doce, Antonio Méndez Rubio, Agustín Fernández Mallo, Melcion Mateu, Mariano Peyrou, Julieta Valero, Pablo García Casado, José Luis Rey, María Do Cebreiro, Sandra Santana et Juan Andrés García Román. 


\section{L'écriture fractale ou l'objet de la simultanéité de Ramón Dachs}

L'écriture géométrique n'est assurément pas d'emblée une écriture poétique. L'écriture fractale est une écriture de l'extension. Chaque élément s'y trouve reproduit à une échelle différente. Pour Ramón Dachs cette écriture géométrique pourra permettre d'accéder à un système de pensée où plusieurs plans convergent. Depuis l'âge de dix-neuf ans, Dachs travaille à l'élaboration de son écriture géométrique et fractale. Dès l'année 1978 et, par la suite en 1980 et 1982, par des expositions collectives à Barcelone le poète met en place et développe son projet d'écriture géométrique. Des écritures «1, 2 et 3 » de 1978, il passe aux écritures « 4 et 5 » en 1989 et à la $6^{e}$ hyperspatiale en 1993. Il faudra attendre le 28 septembre 1998 pour que le poète inaugure son écriture fractale lors d'une exposition à l'IVAM. Bien qu'il ne la considère pas comme de la poésie, Dachs publie son œuvre dans des collections de poésie. Il est d'ailleurs reconnu comme poète. Il est clair que l'usage que prétend faire le poète de ses fractales n'est pas qu'un jeu visuel mais bien une structuration géométrique se substituant à la syntaxe habituelle. S'il refuse cependant pour ses textes l'appellation de poésie visuelle, c'est que ses recherches sur l'espace et les possibilités de dire simultanément plusieurs éléments, plans et niveaux, dépassent la simple disposition visuelle: il s'agit pour lui d'y inscrire une pensée aux multiples connexions et interconnexions. Dachs déploie toutes les possibilités de la géométrie. Il trouve ses modèles parmi des écrivains comme Ramon Llull, Bruno et Leibnitz, ou encore les cabalistes et les soufistes. Le mysticisme présent dans ces pensées l'est aussi dans l'œuvre de Ramon Llull (1232- 1315). Le recueil Codex Mundi (2012) de Dachs s'ouvre avec le Taula general (1293-1294) du philosophe et poète Llull et ses possibilités géométriques. La représentation des neuf attributs « $\mathrm{B} / \mathrm{K}$ du Créateur» : générosité, grandeur, éternité, pouvoir, sagesse, volonté, vertu, vérité et gloire, est mise en relation binaire avec un tableau de trente-six segments. Dachs voit en Llull le précurseur de l'écriture géométrique, et le Dante catalan. Lorsque le poète ouvre son recueil par ce schéma llulien, il relie directement son écriture fractale à celle de Llull. La syntaxe linéaire est remplacée par une géométrie qui combine des mots et des concepts pour créer un réseau d'interconnexions. Dans Blanc. Topoèmologie (2007), ce sont les langues qu'il combine. L'espagnol, le français et le catalan créent ainsi un réseau de sens, ouvrant, pour chacun des mots, des perspectives et des voies nouvelles par cette contamination et cette juxtaposition de langues. Le mot peut alors emprunter plusieurs chemins.

absoluto fervor

envie du tout

còsmica apetència

(absolute ferveur / envie de tout / cosmique appétence)

Ce jeu poétique de diglossies et de fragmentation du mot par l'inclusion d'italiques densifie le réseau de signification et d'images. Blanc montre comment Dachs s'est orienté vers une écriture géométrique complexe. Dans l'exemple 
que nous venons de reproduire, aucune figure géométrique n'est utilisée mais les italiques déconstruisent les mots pour en élaborer de nouveaux. Les mots sont interconnectés entre eux et avec ceux préfigurant la déconstruction. "sol», «vie» et «còs» sont les trois mots qui émergent de ce jeu typographique: «soleil», «vie» et «corps». On peut ainsi lire le poème dans la linéarité de ces vers, sans tenir compte des changements typographiques, mais on peut aussi reconstruire de nouvelles combinaisons de mots selon le critère de la langue ou de la typographie. Ces trois vers, trois syntagmes nominaux, s'adonnent ainsi dans toute la complexité et la densité de ces combinaisons plurielles.

À chaque langue est associé un univers différent: le mysticisme et la passion à l'espagnole ( «absoluto fervor»), la vitalité, l'inscription dans la moralité de la vie («envie du tout») pour le français, l'ouverture sur l'univers, la science pour le catalan ( "còsmica apetència»). "sol / vie / còs" relient les trois vers entre eux mais aussi les trois langues, «le soleil, la vie et le corps». La diglossie offre un jeu de codage où chaque langue est une clé à un sens supérieur du poème.

ab. to. fervor

en - du tout mica - ape

Le poète joue des sonorités renvoyant au système phonologique et à ses tableaux résumant les combinaisons sur l'axe paradigmatique: les occlusives $a b$ [b] / ape [p], les voyelles to / tout. Le poète joue encore avec de nouveaux mots et sens: $\underline{\text { mica, }}$ terme relevant significativement de la géologie et provenant du latin, mica, «miette». Ce minéral a reçu ce nom en raison de sa texture feuilletée. N'est-ce pas la texture de ces vers? Et, du cosmos à la miette, ne sommesnous pas dans ce que cette poésie tente d'exprimer? "Du tout» isolé par le jeu typographique ne dit-il pas exactement le contraire de ce que dit le vers lu sans tenir compte de sa fragmentation par l'italique? "du tout» renvoyant à "pas du tout». Un sens et son contraire dans un même vers. Le tout et le rien. Par ailleurs, comme dans le poème «Le dormeur du Val» (1870) d'Arthur Rimbaud, le vers nous invite à décomposer l'adjectif: "absoluto", sol et luto, c'est-à-dire le soleil et le deuil, la vie et la mort. "absoluto" fonctionne ici comme oxymore. Le poète fabrique ainsi une poésie fractale sur la banquise de la page blanche, ce qu'il poursuivra dans son cycle Euràsia/Transeurasia/Antarctique (1978-2008) qui s'achève par un voyage- performance en Antarctique. Perte de la linéarité du poème et déploiement par les fractales d'une infinité d'écritures et de lectures, voilà ce que Dachs élabore dans ses propositions poétiques et quantiques.

L'écriture géométrique de Dachs s'inscrit, d'une part, dans la continuité de la poésie concrète exposée dans les musées et, d'autre part, dans son travail sur la présence et l'absence. Dachs se lance dans la composition d'une poésie qui s'auto-génère à partir de différentes écritures: "écriture fractale I: la symétrie", "écriture fractale II : le segment», "écriture fractale III : les triangles équilatéraux", "écriture 4 : tétraèdre régulier», "écriture 5 : centre de gravité » et «écriture 6 : intersection orbitale». Le poème en tant qu'objet fractal se caractérise par une construction non- linéaire à partir de l'itération de nombres complexes, 
comme l'a mis en évidence le mathématicien Benoît Mandelbrot. Dachs élabore son écriture poétique sur ce modèle fractal, par l'intertextualité (Quadern rimbaldia pour la présence de Rimbaud, 1996), l'hypertexte (Intermínims de navegació poètica, premier recueil de poésie hyertextuelle publié en Espagne en 1996), le multilinguisme (Blanc. Topoèmologie en espagnol, français et catalan, 2007 pour la version intégrale), le texte collectif diachronique par usurpation (Cent un juejus de Xina Tang, 1997) et la segmentation d'énoncés simultanés (Tot i res Monosticha Catonis, 1999).

«L'écriture géométrique 1 » ou «écriture fractale 1 »s'appuie sur la symétrie. Les mots sont des points dans l'espace et s'articulent dans un cercle. Il y a un jeu de yin et yang, c'est-à-dire ici de dualité entre deux «mots-points ${ }^{9}$ » qui se mélangent. Dans «Crepúsculo» (2012: 21), les mots «día» et «noche» sont les deux points à partir desquels vont pouvoir se confondre et se mêler ces deux éléments. Le cercle est composé d'une phrase qui vient donner un sens théorique à la composition. L'écriture géométrique permet de rendre les mots indéfinis, autrement dit si le jour et la nuit s'opposent aussi simplement que le noir et le blanc, la proposition d' «écriture fractale I symétrique» ouvre ces contraires à une dualité, à une teinte de gris où lumière et obscurité se rejoignent. Dans «Litoral» (21), le même procédé est employé avec les mots «lago» et «isla». Comme l'indique Dachs, un lac est une île d'eau sur terre et une île, un lac de terre sur l'eau. Donc le cercle dans cette composition dessine les contours de l'île qui englobe les deux mots. Chacun de ces deux mots contenant quelque chose de l'autre, c'est le duel du yin et du yang qui est ainsi représenté. Chacun existe dans l'autre, la suppression de l'un entraîne la perte de sens pour l'autre. Cette circularité insulaire dit aussi le refus de la linéarité de la syntaxe. À la linéarité il préfère la simultanéité que permet la "géométrie» ou plutôt l'irréductibilité fractale.

«L'écriture géométrique 2 » ou «écriture fractale 2 » se construit par un dispositif en segment dans une connexion unidimensionnelle. La lecture se fait d'un point (début) à un autre point (fin) dans un système fermé. Dachs propose une rupture de la linéarité de la syntaxe par la fractalité qui amène la simultanéité. Pourtant avec le segment fermé, il fait le choix d'une perte de synchronie. La lecture va du point A au point B. Il n'y a plus de mélange mais un cheminement du premier «mot-point» au second «mot-point». Deux dispositifs spatiauxtemporels incompatibles sont combinés dans un oxymore fractal.

«L'écriture géométrique 3 », dite également «écriture fractale 3 », multiplie, pour sa part, les possibilités par une composition qui s'appuie sur douze triangles équilatéraux, permettant, cette fois, une connexion bidimensionnelle. La symétrie est visible par le jeu de miroir des six "mots-points», chacun reproduit deux fois. Les douze «mots-points» forment une étoile: chaque mot est interconnecté à deux autres mots dans un triangle équilatéral.

9 Le terme «palabra-punto » est employé par Dachs. 
«L'écriture fractale $3 »$ reprend les techniques des écritures « 1 et 2 ». La symétrie y est utilisée dans le positionnement des "mots-points» en étoile et en triangles équilatéraux. L'ordre se dégage du désordre apparent des points. Nous retrouvons par ailleurs la limite du segment propre à «l'écriture 2 » dans la symétrie des angles de «l'écriture 4 », alors que dans «l'écriture 1 », les «motspoints » partagent des éléments communs. Ici, le partage est établi par la somme des angles. La relation entre certains mots est empêchée dans ce dispositif. Par contre une telle écriture propose la superposition des lectures et des sens, et donc une rupture forte de la linéarité. Elle propose des probabilités infinies de sens et de combinaisons.

Si la superposition de la composition commence avec «l'écriture fractale 4 », "l'écriture 5» apporte une dimension supplémentaire: elle repose sur le tétraèdre régulier formé de quatre triangles équilatéraux identiques («écriture $3 »)$ reliés par six arrêtes à quatre sommets. La fonction tridimensionnelle génère une connexion avec un quatrième «mot-point», tout en conservant aussi la stricte délimitation dans le segment («écriture 2 »). Dans «Reproducción» (31), les "mots-points» sont "homme», «femme» et "accouplement». Ils convergent vers le «mot-point»: "descendance». Synthèse des écritures «1, 2 et 3 », «l'écriture 4 » introduit cependant une nouvelle dimension, c'est-à-dire le quatrième sommet qui donne une tridimensionnalité à la construction poétique : dans "Reproducción", par exemple, en intégrant le mot "descendance » à la fois comme un point de fuite et comme une insertion nouvelle dans l'espace.

Dans «l'écriture fractale 5 » c'est le centre de gravité qui est représenté comme l'unique "mot-point» de la construction. Le poème, épuré de sa construction complexe en tétraèdre et donc des écritures antérieures, ne laisse voir plus qu'un seul «mot-point», noyau du poème et du sens.

Les quatre premières "écritures fractales ou géométriques» ont permis de construire une relation d'interconnexion en quatre points dans un espace tridimensionnel. "L'écriture fractale 5 », pour sa part, efface les traits de construction pour ne laisser visible que le centre.

«L'écriture 6 » apportela 4 e dimension géométrique, c'est-à-direle mouvement. Le mot se dédouble à partir d'un nom et surtout d'un verbe au gérondif. Le point du poème "Madurez» (35) est le syntagme verbal «envejecer fecundando" qui joue de la dualité propre à «l'écriture 1 ». Double dualité d'emblée si nous considérons le titre: 1 . vieillir/mûrir 2. mûrir/créer («fecundando»). La maturité annoncée par le titre du poème se trouve ici évoquée dans une double relation entre le vieillissement et l'acte créatif. Mûrir est la condition pour créer, mais en retour créer est aussi ce qui produit la maturité. Le poème "Summum» (34) fait un choix différent avec l'utilisation du participe passé "amado»: "amante amado». Pas d'action en cours mais un constat: «l'amant aimé » dans le système du segment fermé est celui qui aime et qui est à la fois aimé en retour. On perd avec le participe passé la relation de cause à effet introduit par le gérondif dans le premier exemple. Ce n'est plus le «mot-point» qui prévaut mais la «locution 
verbale-point» qui intègre les six écritures fractales. L'écriture fractale poétique élargit la relation entre poésie et science. Mots et chiffres ne sont plus dans un dialogue mais dans une mutation. La géométrie permet la construction d'un système sémantique et graphique dédoublant les lectures et les interprétations. Il ne s'agit plus de se servir des mécanismes de la poésie pour la mnémonique scientifique ou de références scientifiques afin de provoquer des métaphores, mais de construire une poésie et une science qui feraient sens l'une avec l'autre. C'est en cela que l'utilisation des fractales parvient à produire des «métaphores scientifiques» et des "équations poétiques». Jesús Malia (2011: 224) précise: «la metáfora es la aritmética elemental de la poesía» («la métaphore est l’arithmétique élémentaire de la poésie»).

Ainsi, la poésie espagnole actuelle à travers ses propostions "postpoétiques » et fractales remet en cause la relation au temps et à l'espace par la production de systèmes en réseaux et nœuds: fusion des genres poétiques ou non (multivers), dissociation aux formes antérieures, détournement du lieu de la poésie (topos trompeur). Le «système postpoétique» se dissocie du "système orthodoxe» selon les critères d'Agustín Fernández Mallo qui met en évidence les attracteurs "postpoétiques» dans une zone instable et chaotique, alors que la "poésie orthodoxe» repose sur des attracteurs dans une zone stable et non-chaotique. Les "postpoèmes» s'articulent par des boucles de rétroaction qui assimilent des informations étrangères à la poésie, telles les publicités ou les sciences, mais contaminent aussi ces données produisant alors des excroissances poétiques. Cette tentative de reproduction sur un plan horizontal, comme les plantes adventives ou rhizome "postpoétique», est identifiable dans la poésie fractale de Ramón Dachs dont les écritures géométriques répondent aux recherches de Benoît Mandelbrot. Les tentations de la nouvelle poésie espagnole, identifiée comme «Poétique des singularités» par Vicente Luis Mora, sont à l'inverse de la «Poésie de la normalité», une poésie déplacée, inattendue et étrange.

Anormales ou accidentelles, ces boutures poétiques explorent les ressources de l'espace par leur présence dans les musées, les recherches mathématiques ou les médias numériques. Le lecteur est alors transformé ainsi que le temps de la lecture. Qui lit et qui regarde? Le poème se met en action face à un lecteur-spectateur à la fois voyeur et protagoniste. Si cette poésie-caméleon les «postpoètes sont souvent affiliés à la "Génération mutante» - expérimente toutes les formes de la création contemporaine, elle porte en son sein les empreintes et souvenirs du temps.

\section{Bibliographie}

Bailly, J-C, 2015, L'élargissement du poème, Paris, Christian Bourgois Éditeur, coll. «Détroits». Bonnargent, É., 2011, Atopia, petit observatoire de littérature décalée, Lyon, Le Vampire Actif, coll. «Les Entretiens».

Borges, J.L. (1960), 1997, El hacedor, Alianza Editorial, Madrid. 
Claass, A., 2014, Du temps dans la photographie, Trézélan, Filigranes Éditions.

Dachs, R., 2007, Blanc. Topoèmologie, Reims, Le clou dans le fer, coll. « expériences poétiques».

Dachs, R., 2012, Codex Mundi, Madrid, Amargord Ediciones, coll. "pi de poesía ", N². Fernández Mallo, A., 2004, Creta Lateral Travelling, Palma de Majorque, Sloper, coll. «La noche polar».

Fernández Mallo, A., 2009, Postpoesía. Hacia un nuevo paradigma, Barcelone, Anagrama, coll. «Argumentos».

Lê, L., 1999, «Littérature déplacée» in: Tu écriras sur le bonheur, Paris, PUF, coll. «Perspectives critiques».

Malia, J. (dir.), 2011, roetas. Primera antología de poesía con matemáticas, Madrid, Amargord Ediciones, coll. "pi de poesía».

Mora, V.L., 2006, Singularidades. Ética y poética de la literatura española actual, Madrid, Bartleby Editores, coll. «Miradas».

Pascal, B. (1896), 2004, Les Provinciales, Pensées et opuscules divers, Paris, Éditions Garnier, coll. "La Pochotèque».

Portela, A., 2003, ¿Estás seguro de que no nos siguen?, Barcelone, DVD Ediciones, coll. «poesía».

Sartre, J-P. (1946), 1996, L'existentialisme est un humanisme, Paris, Folio, coll. «Essais». Tisselli, E., 2010, El drama del lavaplatos, Salamanque, Editorial Delirio, coll. «Krámpack, 8 ». 